\title{
URUGUAY COMO ESTADO PEQUEÑO EN EL MERCOSUR (1991-2020): UNA LECTURA DESDE LA AUTONOMÍA REGIONAL ${ }^{1}$
}

\author{
Camilo López Buriana \\ aProfesor Adjunto en la Universidad de la República (Ciencia Política-Facultad de Ciencias \\ Sociales y Relaciones Internacionales-Facultad de Derecho), Montevideo, Uruguay, Integra el \\ Sistema Nacional de Investigadores (ANII).E-mail: camilo.lopez@cienciassociales.edu.uy \\ Orcid: 0000-0003-1185-854X \\ María Cecilia Míguez
} bInvestigadora Adjunta de CONICET. Vice directora del Instituto de Investigaciones Históricas Económicas Sociales e Internacionales (IDEHESI), docente de grado y posgrado. Universidad de Buenos Aires, Argentina.E-mail:mmccmiguez@gmail.com Orcid: 0000-0001-7875-0280 http://dx.doi.org/10.1590/0102-181216/112

\section{Introducción}

Este artículo analiza el devenir de Uruguay en el proyecto Mercado Común del Sur (Mercosur) desde 1991, año de la firma del Tratado de Asunción, hasta la actualidad por medio de un breve recorrido por estos años de vigencia del proceso de integración, buscando periodizarlo en distintas etapas, teniendo en cuenta como eje central la intención de los proyectos políticos locales de construir un espacio de autonomía regional. Para ello, dialogamos con una serie de perspectivas teóricas que analizaron el concepto y la práctica

\footnotetext{
1 Este trabajo forma parte de los resultados del Proyecto I+D "La política exterior uruguaya entre la autonomía y el alineamiento (1946-2019): Factores sistémicos y domésticos en la explicación de una trayectoria", financiado por la Comisión Sectorial de Investigación Científica (CSIC) de la Universidad de la República (Uruguay).
} 
de autonomía en el continente pero atendiendo a las particularidades de este concepto para el caso de los Estados pequeños como el Uruguay.

En 2021 el proceso de integración regional del Cono Sur -que se instituyó por la creación del Mercosur- cumple treinta años de existencia en un contexto particular de crisis. Si bien evidencia una continuidad más allá de los recambios gubernamentales, dicha continuidad no implica que haya habido un proyecto lineal, ni exento de las grandes contradicciones que signaron los procesos económicos, sociales y políticos internos en estas tres décadas. Desde sus inicios en 1991 y en clave regionalismo abierto, el Mercosur fue pensado como plataforma de ingreso a la globalización neoliberal. Así lo prueban no solamente sus condiciones de gestación, sino también los tempranos acuerdos con los Estados Unidos (acuerdo 4+1, los cuatro países del Mercosur en bloque) y con Europa.

A diferencia de esta etapa caracterizada por el auge de las políticas neoliberales, el siglo XXI se caracterizó en América Latina por la emergencia de gobiernos que cuestionaron en distinto grado y medida los pilares ese orden neoliberal. Un regionalismo suramericano acompañó ese proceso, marcado por una "dinámica a la carta" y la primacía de las agendas sociales y políticas sobre las comerciales (Quiliconi y Salgado Espinoza, 2017; Sanahuja, 2014). El Mercosur se constituyó como un espacio de articulación regional en sentido amplio, donde fue posible disminuir la injerencia de potencias mundiales y construir agencia local. Por esta razón, creemos relevante periodizar la participación de Uruguay en el Mercosur teniendo en cuenta esta perspectiva de búsqueda de autonomía, la tradición a la que se remonta y las posibilidades y particularidades para el caso de un Estado pequeño.

Consideramos que la noción de autonomía encierra una reivindicación histórica en la región que, por oleadas, 
se reaviva en diversas fuerzas políticas que se expresan en gobiernos nacionales. Así como la integración regional constituye un tópico central en los países del continente (Quiliconi y Rivera, 2019), la autonomía continúa siendo una referencia recurrente en las reflexiones sobre la inserción internacional. Son conceptos que están vinculados. El devenir y el desarrollo pendular de los regionalismos también se relaciona con las motivaciones aperturistas o autonómicas en los países latinoamericanos (Tussie, 2009). Tal como afirma Deciancio (2016), el regionalismo surgió en las Relaciones Internacionales de América Latina como la reflexión sobre las posibilidades y los desafíos del desarrollo y la autonomía.

En América Latina, el regionalismo posneoliberal (Sader, 2013) persiguió una serie concreta de objetivos:

[...] Revalorizar la capacidad reguladora del Estado; mayor capacidad de negociación frente a la apertura y la liberalización comercial y financiera; cuestionar el modelo económico dominante; situar el tema de la justicia social y la redistribución del ingreso y actualizar el pacto social mediante nuevas relaciones entre movimientos sociales y gobierno. (Preciado Coronado, 2014, p. 47)

La autonomía volvió al centro de la cuestión, esta vez como estrategia y como meta, con el objetivo de alejarse de las dinámicas impuestas por el Consenso de Washington y de las "recomendaciones" del Fondo Monetario Internacional (FMI). Ese regionalismo condensado en nuevos organismos como la Unión de Naciones Suramericanas (Unasur) o la Comunidad de Estados Latinoamericanos y Caribeños $(\text { Celac })^{2}$-así como la propia resignificación del

\footnotetext{
2 La Celac representa un parteaguas en la historia latinoamericana, pues por primera vez se reunieron Jefes de Estado y de Gobierno, sin la tutela de potencia extranjera alguna (Preciado Coronado, 2014).
} 
Mercosur- demostró que es posible pensar una autonomía regional, que potencie los desarrollos nacionales locales. Un espacio de construcción colectiva que permite márgenes de acción para cada una de las naciones que la conforman, impulsando, además, sus demandas históricas en el sistema internacional.

Así veremos que en estos treinta años, los gobiernos del Frente Amplio (FA) apelaron a esta tradición ideológica y a esta cosmovisión de la autonomía, a diferencia tanto de sus antecesores como del gobierno actual de Luis Lacalle Pou. Analizaremos entonces cómo aparece la apelación a la región en las distintas etapas y qué rol cumple o aparenta cumplir para las distintas fuerzas políticas. Por último, avanzaremos en la definición del concepto de autonomía regional.

\section{Puntos de partida: autonomía, identidades, agencia y estructura}

El concepto de autonomía en América Latina ha sido especialmente desarrollado en Brasil, en Argentina y en Colombia. ${ }^{3}$ Inicialmente, las corrientes que lo abordaron reflexionaron acertadamente sobre las posibilidades de desplegar una política exterior no necesariamente alineada con las propuestas de las potencias internacionales, $\mathrm{y}$ asociaron el accionar en el plano global con necesidades económicas, sociales y políticas internas. Cuestionaron las asimetrías de poder en el plano mundial pensando mejores opciones para los países periféricos. Entre las estrategias que plantearon como válidas están presentes la integración regional y los distintos modos de coordinación de las acciones políticas. En la literatura clásica de la Teoría de la Dependencia, los vínculos entre autonomía e integración

\footnotetext{
3 Ver por ejemplo la conceptualización de autonomía periférica de Drekonja Kornat (1983) y desde una óptica estructuralista el trabajo de Cepeda Ulloa (1986).
} 
fueron tratados -tanto por Helio Jaguaribe como por Juan Carlos Puig- como de tipo instrumental. En ambos casos, la integración es pensada al servicio de la autonomía como una dimensión agregada y una de sus principales funciones consistiría en operar como "reaseguro" o "garantía" de los cursos de acción autonomizantes (Simonoff y Lorenzini, 2019). En este trabajo, y pensando en las características de un Estado pequeño, afirmaremos que ambas nociones pueden ser entendidas como coconstitutivas y, por lo tanto, condicionarse mutuamente.

Nuestro enfoque tiene diferentes afluentes, las Teorías de la Dependencia, los desarrollos de Robert Cox y las corrientes de la Economía Política Internacional, y finalmente los aportes de uno de los componentes del denominado "cuarto debate" de las Relaciones Internacionales: la mutua interacción y constitución entre agencia y estructura, atendiendo a sus componentes materiales, institucionales e ideacionales (Sanahuja, 2018, p. 108).

Pensar el dilema de la autonomía para Uruguay, en cuanto Estado pequeño, implica abordar uno de los puntos señalados como de mayor relevancia para la literatura más reciente sobre este tipo de actores internacionales (Baldacchino y Wivel, 2020, p. 9). Y pensarlo críticamente implica asumirlo desde una perspectiva reflectivista, que desde el punto de vista analítico parte de la naturaleza esencialmente contingente, histórica, contextual e intersubjetiva del conocimiento (Sanahuja, 2018, p. 108), reconociendo -en consecuencia- su carácter performativo (Perrotta y Porcelli, 2019, p. 210).

Tal como ha analizado Bernal-Meza, la autonomía ha sido abordada en la región como concepto, como herramienta metodológica y como estrategia (2016). Proponemos en este trabajo que, dado su base en una epistemología reflectivista, el enfoque autonomista forma parte del conjunto de teorías constitutivas. Estas tienen en común el 
propósito de dar cuenta de la naturaleza de las relaciones internacionales a partir de las estructuras que las constituyen y condicionan. Como señala Sanahuja:

Las teorías constitutivas entienden las teorías de las Relaciones Internacionales como elementos que constituyen la realidad social a través del conocimiento que estas generan, y de las instituciones, las prácticas sociales, el lenguaje y los discursos y narrativas a las que ese conocimiento daría forma. Para estas teorías el conocimiento está enraizado en las relaciones sociales y es parte de ellas. Tiene un papel constitutivo de esa misma realidad, en tanto otorga significado a las relaciones sociales y orienta las prácticas de los actores sociales, construyendo el mundo conforme a la comprensión e interpretación del mismo por parte de esos actores. (Sanahuja, 2018, p. 108)

Por otra parte, frente a los discursos performativos de la anarquía (Wendt, 1992), la idea de autonomía se posiciona como una contribución local crítica a los estudios internacionales, ya que cuestiona los supuestos del mainstream (Míguez y Deciancio, 2016, p. 186).

Un significado posible para la autonomía puede ser la determinada capacidad del Estado para diagramar e implementar determinadas decisiones en forma relativamente independiente de los diversos actores sociales locales e internacionales, fijar metas y alcanzarlas de forma independiente. Sin embargo, aquí incluimos lo que consideramos el sentido histórico inicial del concepto como condensación de prácticas concretas (Palti, 2007), alejándonos de las visiones realistas que la consideran un objetivo válido para todos los Estados. Es decir, no es solo una capacidad y una disposición, sino que encierra un objetivo con un contenido histórico particular: la discusión de las asimetrías del orden mundial en función de la protección de la soberanía 
nacional o regional. Tomassini (1989) definió a la autonomía como "un objetivo de las políticas que podría alcanzarse en forma gradual y creciente mezclándose, en dosis variables, con situaciones de dependencia, interdependencia y condicionamiento externo" (p. 302). Es decir, no es solo un instrumento, sino también un objetivo vinculado con el interés nacional.

Como es sabido, quien primero volcó el concepto en el área de las Relaciones Internacionales fue el intelectual brasileño Hélio Jaguaribe en el trabajo titulado "Dependencia y autonomía en América Latina” (1969), en que desarrolló los fundamentos del concepto y un Modelo Autónomo de Desarrollo e Integración de América Latina (p. 66). Luego, en 1979, publica otro trabajo en esta línea: "Autonomía periférica y hegemonía céntrica”, en el cual diferenció cuatro niveles de estratificación en el sistema internacional resultante de la Segunda Guerra Mundial: las naciones con primacía general, con primacía regional, con autonomía -es decir, margen de autodeterminación en asuntos domésticos y capacidad de actuación internacional independiente-, y las dependientes (mayoritarias), cuya actuación se encuentra condicionada por factores y decisiones externos (Jaguaribe, 1979).

Para Jaguaribe, los espacios autonomistas solo son posibles mediante la viabilidad nacional de un país periférico y la permisibilidad internacional. En este sentido, encontraba en la existencia de recursos humanos y materiales adecuados, en el grado de cohesión sociocultural y en la capacidad de neutralizar las amenazas externas, las condiciones para la consecución de la autonomía. Agregó desde sus primeros trabajos la necesidad de la autonomía tecnológica y empresarial (Jaguaribe, 1979, pp. 96-97).

En la Argentina, Puig elaboró de forma sistemática un modelo para comprender la política exterior argentina en 
clave similar. ${ }^{4}$ A lo largo de sus trabajos fue esbozando lo que se ha denominado como una "Doctrina de la Autonomía". Para Puig (1980), la autonomía de un Estado es "la máxima capacidad de decisión propia que se puede lograr, teniendo en cuenta los condicionamientos objetivos del mundo real" (p. 145), y depende la lectura del sistema internacional que hagan 'élites funcionales' a un proyecto autonómico.

Desde los inicios, tanto Jaguaribe como Puig dieron lugar a la integración regional como un elemento funcional a la consecución de la autonomía. ${ }^{5}$ Jaguaribe planteó la necesidad de aprovechar espacios de permisibilidad en el sistema mundial, relacionando esas posibilidades con la mejora en la escala de producción y la productividad -bases del desarrollo- por medio de la integración regional (Jaguaribe, 1982, p. 4). Es decir, la autonomía no constituye solamente un elemento de capacidad decisional, sino que representa el ejercicio de construcción de fuerzas productivas que habiliten el 188 espacio para la consecución de determinados intereses.

Puig también reflexionó sobre la importancia de la integración, en particular poniendo en tensión distintos proyectos en disputa en el contexto de escritura de sus trabajos. Así fue crítico de las orientaciones "comercialistas" que caracterizaron a la propuesta de la Asociación Latinoamericana de Libre Comercio (ALALC) y al Mercado Común Centroamericano (MCCA). Las consideró funcionales a las expectativas de los Estados Unidos y de las empresas transnacionales, y contradictorias a la consecución de

\footnotetext{
4 Otros trabajos que continuaron estos planteos son los de Miranda (2003, 2005), Bernal-Meza (2005), Bologna (2008), Dallanegra Pedraza (2009), Colacrai (2009), Lechini (2009), y Simonoff (2007, 2010, 2013, 2016), entre otros. Briceño Ruiz y Simonoff (2015, pp. 10-12) agrupan a estos autores dentro de una misma categoría conceptual de autonomía, en que esta se define como capacidad de decisión o acción, diferenciándolos de otros planteos teóricos que conciben la autonomía asociada a la capacidad de negociación (Escudé, 1992, 1995; Russell y Tokatlian, 2001).
}

5 Sobre este punto puede consultarse los trabajos de Briceño $(2012,2015)$. 
autonomía (Puig, 1986). Frente a esta visión su propuesta exaltaba la posibilidad de una integración "solidaria", con predominancia de aspectos políticos culturales: "[...] la integración solidaria se fundamenta en alianzas, vale decir, en uniones de Estados decididos a conseguir determinados objetivos sectoriales, pero que se fundamentan en valores compartidos, entre los que descuella el de autonomía” (Puig, 1986, pp. 59-60).

Ahora bien, ¿en qué sentido sigue siendo válido apelar a la autonomía como objetivo y estrategia, incluso en el caso de un Estado pequeño, contra los pronósticos desalentadores de mainstream que alimentan inserciones internacionales asociadas a potencias predominantes para obtener beneficios concretos? A diferencia de la mirada instrumental, propondremos entonces atender al rol de lo regional como aspecto clave para posibilitar márgenes de autonomía para un Estado pequeño como el Uruguay. Volveremos sobre estos aspectos hacia el final del artículo, en nuestra definición de la autonomía regional.

Para interpretar las posiciones de las dirigencias políticas respecto de la región, a lo largo de los últimos treinta años, creemos que es importante remitirnos tanto a los contextos históricos como a las identidades de las élites y su impacto en la política exterior. Siguiendo a Sanahuja (2018), el análisis de las estructuras históricas concretas nos permite comprender la mutua constitución de los actores y la estructura, entendiendo que esa relación da forma a la identidad, los valores y los intereses, así como explican su agencia.

En el siguiente apartado haremos una periodización de la política exterior uruguaya respecto del Mercosur, teniendo en cuenta la apelación a la región que las distintas fuerzas políticas hicieron durante su gestión. A partir de las reflexiones de Acuña y Chudnovsky (2013), consideramos que la capacidad de agencia implica una identidad y, a la vez, supone capacidad de acción estratégica desde los recursos disponibles. La matriz 
identitaria es un punto de referencia para definir los intereses, que se constituyen en estructurantes de los objetivos, para los que se movilizaran recursos y capacidades en el marco de una estrategia fijada. Como señala Pizzorno, los objetivos y necesidades que los actores se proponen varían según las diferentes identidades colectivas en las que se sustentan (1985, pp. 37-38). Veremos que la consideración de la región como espacio de construcción colectiva en clave de autonomía regional fue parte constitutiva de la orientación y la cosmovisión de los gobiernos del FA, aunque su conceptualización no haya sido explícita a nivel programático.

\section{El derrotero de Uruguay en el Mercosur en tres tiempos: alineamiento asimétrico, impulso de la autonomía regional y llegada de la crisis de la globalización neoliberal}

En este apartado, dirigiremos la reflexión hacia nuestra 190 hipótesis principal. Consideramos que es posible identificar dentro del proceso de integración del Mercosur un período de construcción de autonomía regional a la que el Uruguay del FA aportó sobre la base de la convicción ideológica de sus líderes principales y la acumulación histórica de su constitución como fuerza política. Asimismo, observamos que el uso de la noción de autonomía en el caso de un Estado pequeño es distinto que en los casos de países de la región con escalas de desarrollo productivo más amplias como el caso de Argentina y Brasil. Por último, si bien todo proceso de integración o regionalismo implica pérdida de soberanía decisoria en aspectos puntuales, la participación en el ámbito de un espacio más amplio que se caracteriza por la reivindicación y protección del rol de los países periféricos en el sistema mundial potencia la autonomía de un Estado pequeño para mejorar su inserción internacional.

De acuerdo con el análisis del proceso histórico, podemos identificar tres períodos en el derrotero del Mercosur. 
Al primero lo denominaremos de alineamiento asimétrico (1991-c.2003), al segundo lo conceptualizaremos como el impulso de la autonomía regional (c.2003-2015) y al tercero (c.2015-al presente) lo caracterizaremos como un tiempo de crisis de la globalización neoliberal (Sanahuja, 2019).

\section{Los tiempos del alineamiento asimétrico} (1991-c.2003)

Muchos factores contribuyeron al surgimiento del Mercosur, y esta temática ha sido ampliamente abordada por la literatura de las relaciones internacionales en la región. Hay que recordar que la década de 1980 se inicia con la firma de un nuevo tratado ante el acuciante fracaso de la ALALC, el Tratado de Montevideo que dio origen a la ALADI. ${ }^{6}$ Ese será el marco en el que los gobiernos de Raúl Alfonsín en la Argentina y José Sarney en Brasil relanzarían el proceso de integración regional. Según Roberto Russel,

la mayor complejidad y fluidez del orden mundial, la creciente independencia entre las naciones desarrolladas, el fenómeno de la difusión del poder, la formación de alianzas y el desarrollo de negociaciones por áreas temáticas, entre otros factores de peso, habían dejado al Sur casi a la deriva y profundizado su incapacidad estructural para articular y organizar eficazmente la dimensión correspondiente a sus coincidencias objetivas. (Russell, 1994, p. 8).

Estas circunstancias habrían propiciado la firma de los primeros acuerdos en el marco de los nacientes gobiernos democráticos. Además, las recientemente recuperadas democracias requerían fortalecerse y consolidarse, en el marco de la política de Washington que, aunque con

\footnotetext{
6 Creada en 1960 con el propósito de construir una zona de libre comercio, fue sustituida en 1980 por la Asociación Latinoamericana de Integración (ALADI) cuyo objetivo final busca el desarrollo de un mercado común.
} 
claroscuros a nivel latinoamericano, apoyaba la retirada de las dictaduras en la región. Los primeros protocolos firmados entre Argentina y Brasil reflejaban la decisión de unificar espacios económicos, mayor autonomía e independencia en relación con el mercado mundial (Moniz Bandeira, 2002, p. 32). Esas intenciones serían prontamente reemplazadas por el impulso liberalizador de Consenso de Washington. El Acta de Buenos Aires de julio de 1990, firmada por los presidentes Fernando Collor de Mello y Carlos Saúl Menem, refrendó los acuerdos anteriores e impuso la desgravación acelerada, de forma lineal y automática de los aranceles.

En 1991, se firmó el Tratado de Asunción, incorporando a Uruguay y Paraguay, que fijó la fecha de conformación del Mercosur para el 31 de diciembre de 1994. Como es sabido, el modelo Mercosur se vio impulsado por metas diferentes a las que había motivado a los demás proyectos integracionistas. Especialmente, fue convirtiéndose en un medio para 192 la integración a la economía globalizada y, por lo tanto, un espacio económico disputado por las grandes potencias: los Estados Unidos y Europa. La progresiva apertura de las economías nacionales y su orientación hacia el mercado mundial repercutieron imponiendo prioridades como la orientación hacia el mercado mundial y el fortalecimiento de los mecanismos de mercado (Míguez, 2013).

En paralelo Uruguay, tanto en dictadura como luego en democracia desde 1985, contó con élites que constituyeron coaliciones de política exterior que reaccionaron rápidamente para sumarse, proactivamente, a los procesos bilaterales de Argentina y Brasil, impulsando también la incorporación de Paraguay (López Burian, 2015). El clima liberal de época y su influencia sobre los gobiernos, la competencia política en Uruguay y los nuevos formatos coalicionales de gobierno contribuyeron a un acuerdo entre el Partido Nacional (PN) y el Partido Colorado (PC) -ubicados en la centroderecha del espectro político-sobre la orientación de 
la política exterior (López Burian, 2017). Como señalara el primer ministro de Relaciones Exteriores del gobierno del nacionalista Luis Alberto Lacalle (1990-1995), Héctor Gros Espiell: “[...] aunque hay que bregar por una integración latinoamericana más estrecha, ningún latinoamericano debe coartar el universalismo de la política exterior uruguaya." (El Día, 23 abr. 1990 citado en Luján, 1993, p. 62).

Una apuesta a un regionalismo abierto, más abierto que regional, fue la orientación de consenso a partir de las preferencias del PC y el PN. No fue una política exterior acordada entre todas las fuerzas políticas, sino una decisión consensuada entre colorados y nacionalistas, ya que de cierto modo se excluyó a la izquierda. Tal como afirmábamos, esta orientación de política exterior tuvo como objetivo prioritario el relacionamiento con Estados Unidos y la Unión Europea a partir de una concepción instrumental de la región como plataforma, dada la condición de Estado pequeño de Uruguay (López Burian, 2017).

Durante el gobierno de Jorge Batlle (2000-2005), la lectura de una región en crisis la concibió más un "cerco" que una plataforma. "Soltar amarras de la región” se transformó en la hoja de ruta y el norte fue un mayor acercamiento posible a Estados Unidos, principal socio comercial del país por ese entonces (López Burian, 2017).

Tanto el PC en 1985 con un gobierno de partido como los tres gobiernos posteriores de coalición del PN y el PC se ubicaron en la centroderecha. Los actores corporativos que constelaron en su entorno fueron demandantes de apertura y liberalización. La forma de concebir el desarrollo del PN y el PG fue convergiendo con el tiempo. La inserción económica internacional y la política exterior de estas élites uruguayas fueron consensuándose en el marco de un contexto liberalizante y de creciente importancia del relacionamiento económico con los Estados Unidos y Europa. 
La construcción regional buscó tener la utilidad de una plataforma, por lo cual el proceso de integración se redujo uniaxialmente a lo económico-comercial. El Mercosur era un espacio concebido para proyectarse mediante acuerdos de libre comercio con la Unión Europea y con Estados Unidos.

Durante la presidencia de Batlle, el énfasis en estrechar los lazos con Estados Unidos fue la estrategia predominante, en un escenario de crisis, rompiendo incluso con la idea de un Mercosur como plataforma. La agenda de la política exterior, ocupada al inicio del período en recolocar a Uruguay en el mundo luego del proceso dictatorial, fue tornándose eminentemente comercial durante la década de 1990 y los albores del siglo XXI. De esta manera, en 2013, un senador nacionalista ${ }^{7}$ comenta el derrotero de la política exterior del Uruguay desde la vuelta a la democracia en 1985:

[...] Mercosur [...] es una política de Estado, [...] el quiebre con la política de Estado se produce con la crisis del 2002, o sea con la crisis de los países y del Mercosur. [...] La política exterior de Jorge Batlle a partir de ese momento no es una política consensuada. [...] Pero el Frente Amplio está en la etapa del Mercosur ideológico, que no es de Estado. Empieza en el primer gobierno del Frente Amplio este camino. [...] El Mercosur en los últimos años es un acuerdo en sintonía con el "giro a la izquierda", es un acuerdo ideológico, y esto hace que dentro del país haya divergencias. Luego viene la Unasur y esto es todavía más, porque es un acuerdo mucho más ideológico. (Citado en López Burian, 2015, p. 182)

Bajo estos gobiernos de centroderecha, el proyecto regional mercosuriano estuvo al servicio del aperturismo liberal, y cuando no lo estuvo fue visto como un "cerco" del que debía

7 La persona entrevistada solicitó mantener su identidad reservada. 
buscarse una "escapatoria”. Las salidas rupturistas generaron disensos en un sistema político caracterizado por el gradualismo y los acuerdos; esto, a la vez, ambientó un nuevo consenso entre el PC y el PN, pero ahora sobre la necesidad de flexibilizar el Mercosur. El Uruguay en el Mercosur de la década de 1990 e inicios del siglo XXI quiso ser un Estado comercialista, orientado por élites funcionales a este fin, que se comportaron de manera aquiescente al orden neoliberal de la globalización en tanto estructura histórica. La región como instrumento no fue otra cosa que una plataforma para un alineamiento asimétrico con el mundo desarrollado.

\section{Tiempos del impulso de la autonomía regional (c.2003-2015)}

Si bien la izquierda uruguaya tiene una tradición histórica profunda en política internacional, que busca amalgamar internacionalismo, latinoamericanismo y antimperialismo, la noción de autonomía aparece solamente de manera implícita en su retórica. Sin embargo, consideramos, tal como lo desarrollaremos aquí, que el autonomismo está presente en sus prácticas.

Este segundo período histórico que delimitamos implicó una serie de condiciones de permisividad internacional y de viabilidad para los Estados de la región. En el caso de Uruguay implicó tres aspectos claves. Primero, dada las dinámicas políticas regionales, no quedar atrapado en lazos de dependencia marcadamente asimétrica e irreversible con un actor global como Estados Unidos. Segundo, el contexto regional aunque tumultuoso permitió al Uruguay tener una referencia de pertenencia desde la cual diversificó su inserción internacional. Y por último se posicionó en una región que contaba con un actor relevante del Sur Global, como fue Brasil en los tiempos de Luiz Inácio Lula da Silva, que buscó liderar un proceso de regionalismo posliberal (Sanahuja, 2008) en un contexto de convergencia de 
proyectos progresistas nacionales ${ }^{8}$ que dieron importancia estratégica a los proyectos regionales. Aunque esto último tuvo dificultades y a veces más retórica que concreciones.

El ciclo progresista uruguayo (2005-2020) mostró diferentes momentos en su relación con la integración. Si bien la izquierda uruguaya es mayoritariamente integracionista, algunas posiciones han cuestionado al Mercosur buscando una forma alternativa de lograr grados de libertad sin abandonar el proyecto. Por otra parte, conflictos bilaterales como el que tuvo lugar con Argentina durante el primer gobierno de Tabaré Vázquez (2005-2010) por la instalación en territorio uruguayo de dos plantas de pasta de celulosa sobre el río Uruguay, que divide ambos países, también impactaron negativamente en el relacionamiento regional.

Más allá de estas tensiones, el contexto global y regional ambientó la permisividad internacional, que se conjugó con la capacidad de coordinación política de los gobiernos 196 del Mercosur que delineaba rumbos en documentos como el Consenso de Buenos Aires (2003) o el Acta de Copacabana (2004), bajo el impulso de Néstor Kirchner y Lula. A la vez que se tradujo en posiciones que contribuyeron a cerrar las posibilidades de un alineamiento asimétrico, por ejemplo al consensuar una posición negativa al proyecto estadounidense del Área de Libre Comercio de las Américas (Alca) durante la IV Cumbre de las Américas, celebrada en noviembre de 2005, en Mar del Plata.

Si bien el Uruguay, gobernado por Vázquez, continuó con los diálogos abiertos con Estados Unidos y tuvo sobre la mesa la posibilidad de firmar un tratado de libre comercio, a la interna del partido de gobierno prevaleció la apuesta integracionista. Este rumbo se sustentó en una lectura del desarrollo y la inserción económica del Uruguay que, optando

8 Debe señalarse que solamente de 2008 a 2012 hubo simultáneamente gobiernos progresistas o que discutieron las políticas neoliberales en todos los Estados parte del Mercosur. 
por la integración en contraposición a la apertura, daba a juicio de este núcleo de la izquierda uruguaya más posibilidades de un desarrollo autónomo. Con argumentos de corte neoestructuralistas y neodesarrollistas, sustentaron la importancia de privilegiar estratégicamente la integración regional, pensando en esta a partir del desarrollo de cadenas productivas regionales. Por otra parte, los acuerdos de libre comercio fueron vistos por este núcleo, dados los asuntos que en ellos suelen incluirse, como trabas para el desarrollo y la innovación autónoma (Garcé, 2014a, 2014b; Garcé y López Burian, 2014; López Burian, 2015; Pose, 2018). Esta postura es compartida y apoyada por la central única de trabajadores y varias organizaciones sociales, a la vez que es mayoritaria en el ala izquierda del FA.

Paralelamente a no quedar atrapado en lazos de dependencia asimétrica e irreversible con Estados Unidos, desde su adscripción regional, Uruguay desarrolló un proceso de diversificación de sus relaciones internacionales que no se limitaron al vector de vinculación con países desarrollados, sino que también apuntó a los países y bloques emergentes del Sur global.

Durante el gobierno de José Mujica (2010-2015), la apuesta por la región y el Sur global tuvo un énfasis mayor, especialmente por medio de la búsqueda de un estrechamiento de los lazos con Brasil (Caetano, López Burian y Luján, 2016). Pero la voluntad de profundización comenzó a verse sin posibilidades de concreción dado el escenario externo. La desaceleración del ciclo de las commodities se hacía sentir.

En 2015, Mauricio Macri llegó al gobierno de Argentina lo que implicó un importante giro en la política exterior del país, priorizando socios tradicionales, alineamientos con las principales potencias occidentales, apertura económica y endeudamiento. El gobierno argentino le dio la espalda a la región y a los organismos que habían sido protagónicos en el período anterior. 
Brasil ingresó en un ciclo de crisis que impactó en el gobierno de Dilma Rousseff, quien, desde el inicio de su mandato, mostró una sensiblemente menor vocación por la política internacional en general y regional en particular si la comparamos con su predecesor. El viraje terminaría consolidándose luego del golpe de estado instrumentado como impeachment, y la posterior asunción de Michel Temer en 2016. La región pasaba a tener una dimensión puramente instrumental para Brasil y lo económico-comercial hegemonizaba la agenda. La crisis brasileña, en el marco de una crisis de la globalización, permitió la emergencia de una ultraderecha neopatriota, contestataria del orden liberal internacional (Sanahuja, 2019), que tomó el gobierno luego que la derecha globalista también fuera derrotada.

Poco tiempo después, con Jair Bolsonaro y Mauricio Macri presidiendo Brasil y Argentina, respectivamente, el Uruguay gobernado por la izquierda quedó con poco margen de manio198 bra. El segundo gobierno de Vázquez (2015-2020) se sumó al pragmatismo comercialista del bloque que finalmente terminó de negociar el Acuerdo con la Unión Europea, pero mantuvo señales autonomistas en el plano político que fueron visibles, podría decirse, como gestos de principios. Entre ellos puede destacarse el permanecer en Unasur frente al retiro de sus socios de la subregión, o no plegarse a la alternativa de Prosur o al Grupo de Lima. La permisividad ya no existía y la agencia de la región tenía otro rumbo.

Sin embargo, antes de este escenario, hubo años que se caracterizaron por una práctica de la integración que, sin estar exenta de tensiones, marchas y contramarchas, facilitó la autodeterminación y la autorrealización de Uruguay y de los restantes Estados parte. Con las obvias tensiones de procesos políticos que nunca son lineales, las dinámicas políticas expresaron las contradicciones entre los proyectos nacionales y la concertación y coordinación política regional en el marco de un proyecto mercosuriano que no 
avanzó significativamente hacia andamiajes institucionales supranacionales, pero que permitió mayores márgenes de maniobra internacional. En esta etapa de impulso autonomista, los gobiernos progresistas del Mercosur encarnaron proyectos que si bien cuestionaron aspectos de la globalización no se constituyeron en antiglobalistas, sino que disputaron los significados de esa globalización en pugna en clave autonomista. Uruguay en este contexto se posicionó y se proyectó desde una región, diversificó su inserción y logró no quedar preso bajo formatos de relacionamiento que le generaran un alineamiento asimétrico.

\section{Algunas líneas sobre el tiempo presente}

El tercer período se enmarca en el cuestionamiento de la globalización neoliberal. La crisis de 2008 y el fin del ciclo de las commodities asociada a ella significó el comienzo de un proceso de desaceleración económica que impactó las dinámicas económicas, sociales y políticas de los países latinoamericanos. La expansión económica y los avances sociales fueron sucedidos por la retracción y, con ella, el arribo de una insatisfacción social que repolitizó a sectores sociales activados en el ciclo anterior, pero que ahora se encontraron dispuestos para encarnar una reacción por momentos antipolítica y por momentos opuesta a las políticas impulsadas por los gobiernos progresistas (Sanahuja, 2019). La región tiene, así, a sus propios descontentos. Estos insatisfechos fueron el apoyo para la llegada de las derechas en Argentina, Brasil, Paraguay y Uruguay (Sanahuja y López Burian, 2020).

Aunque un gobierno progresista asumió en Argentina en 2019, el contexto regional y las relaciones intra Mercosur no han logrado aún establecer un sistema de concertación política que oriente un rumbo común. En este escenario, la región se encuentra en el marco de una crisis global de las élites políticas. Mientras el regionalismo está en crisis, una pandemia recorre el planeta. Esta coyuntura crítica puede 
condicionar dinámicas de regionalización, pero el escenario muestra ausencia de liderazgos y proyectos regionales claros.

Mientras tanto, la política exterior uruguaya del gobierno de Lacalle Pou, quien gobierna con una coalición de partidos de centroderecha y derecha, parece dar señales de querer "soltar amarras de la región" o al menos ganar grados de libertad con relación al Mercosur, retomando el discurso de la flexibilización que lejos de ser autonomista deja al país frente a la posibilidad de alineamientos asimétricos. En armonía con esta estrategia, el gobierno uruguayo en 2020 ha tramitado reformas aperturistas y liberalizadoras que disminuyen potencialmente el control nacional sobre sectores económicos estratégicos para el desarrollo productivo; ha fortalecido el discurso de buscar fórmulas de flexibilización del Mercosur, que podrían interpretarse como intensiones de "desacoplarse" de la región, mientras se insiste en dinamizar la agenda comercial externa y buscar negociaciones o ratificaciones de acuerdos a velocidades diferentes con el resto de los socios. A la vez se han aprobado medidas antipáticas con los países vecinos, como las exoneraciones impositivas a extranjeros que han tenido como objetivo a argentinos de alto poder adquisitivo (Hernández Nilson y López Burian, 2020).

El alineamiento con Estados Unidos también ha estado presente tanto en el tema de la crisis venezolana o la reincorporación al Tratado Interamericano de Asistencia Recíproca (TIAR), como en el apoyo al candidato de Donald Trump, Mauricio Claver-Carone, a la presidencia del Banco Interamericano de Desarrollo (BID). Esas acciones, añadidas al acercamiento a China, parecen configurar rumbos que, aunque convergentes con otros países de la región, no se acuerdan en espacios formales de coordinación regional. La articulación directa con potencias mundiales y flujos globales de capital parece ser un objetivo, evitando la 
intermediación de la "plataforma regional” (Hernández Nilson y López Burian, 2020).

En síntesis, la crisis podría estar ambientando un nuevo momento proclive al alineamiento asimétrico y una pérdida de la autonomía regional que, aunque con falencias, se había construido en años anteriores.

\section{La validez de la estrategia y del objetivo de la autonomía regional}

Hemos afirmado en nuestra periodización que la política exterior de los gobiernos del FA apeló a la región en clave autonomista. Esta reflexión amerita desarrollos conceptuales que desplegaremos en este apartado. La discusión sobre la autonomía de los países latinoamericanos continúa vigente. Es un concepto vinculado a la propia génesis de esas naciones y que ha acompañado la historia del siglo XX. Sin embargo, ese rasgo común no anula la heterogeneidad de la región, y las asimetrías existentes entre los países que conforman la región. Por eso queremos abocarnos a la importancia de pensar desde un Estado pequeño, que generalmente no puede impulsar "de dentro hacia fuera" un proyecto de autonomía nacional "exportable", sino que requiere de una construcción conjunta que habilite mayores márgenes para resolver sus propias necesidades locales. En ese sentido, cobra relevancia la noción de autonomía regional que buscamos definir.

Durante la década de 1990, con la consolidación de las tendencias de los desarrollos de la teoría de las Relaciones Internacionales de la década anterior a partir de las nuevas condiciones del escenario mundial de Posguerra Fría, y en el mismo contexto del surgimiento del Mercosur, el concepto de autonomía fue relegado en las publicaciones académicas. Ello se vinculó a la exaltación de la globalización neoliberal como único camino posible, la afirmación de una supuesta unipolaridad con predominancia de los Estados 
Unidos, e incluso la del fin de los Estados nación. Se predicó la desvalorización de los principios de soberanía y autodeterminación nacional.

Correlativamente se acentuaron los apremios económicos y financieros y el intervencionismo de las grandes potencias hacia las naciones débiles o dependientes, cuya gravitación en el escenario mundial se debilitó notoriamente. Prontamente se revelaron ilusorias las expectativas sobre la conformación de un "poder global", un "gobierno mundial" ejercido igualitaria y armónicamente por instituciones políticas o económicas internacionales como la Organización de las Naciones Unidas (ONU) o el FMI, Banco Mundial (BM), Organización Mundial del Comercio (OMC), o regido sobre la base de acuerdos de las grandes potencias por medio del G-8, el Foro de Davos, la Organización del Tratado del Atlántico Norte (OTAN), etc. En el caso de los países latinoamericanos, con el apogeo del tan mencionado 202 Consenso de Washington, se configuraron modelos económicos que profundizaron la dependencia de la región, que fue escenario de privatizaciones, desregulación, pérdida de poder de decisión por parte del Estado y enorme endeudamiento público. Todo ello se produjo en el marco de la aceptación de los dictámenes de organismos internacionales como el FMI y el BM, ejemplo absolutamente representativo del predominio de las grandes potencias en el escenario de la economía y la política mundial.

Muchos países periféricos, por medio del accionar de sus dirigencias políticas, orientaron su política exterior a la inserción en la globalización, asumiendo, en menor o mayor grado, que la autonomía había dejado de ser un objetivo. Corrientes dentro de la disciplina de las Relaciones Internacionales reformularon el concepto, alejándolo de las condiciones que le habían dado origen y despliegue (Míguez, 2013). Importantes trabajos académicos se orientaron a exaltar los beneficios para los Estados pequeños 
del alineamiento con grandes potencias del sistema, como el caso de los trabajos de Carlos Escudé (1992) y Gerhard Drekonja Kornat (1993). Realizaron una particular lectura de las corrientes neorrealistas, de la interdependencia compleja, incorporando las recomendaciones de Rosecrance para los "estados comercialistas" (1986).

En la Argentina, esta perspectiva tuvo su apogeo durante las gestiones de Carlos Menem (1989-1999) y una reedición en la reciente presidencia de Mauricio Macri (2015-2019) (Míguez, 2017). En Uruguay, irrumpió de forma más nítida durante el gobierno Luis Alberto Lacalle (1990-1995) y se extendió hasta el gobierno de Jorge Batlle Ibañez (20002005). Este tipo de análisis de prácticas que pretendieron mostrarse "pragmáticas" continúan vigentes en la región. En muchas oportunidades, fuerzas políticas conservadoras vuelven a sostener la importancia de "alinearse" con la potencia predominante de turno. En especial en los Estados pequeños, y ante los posibles beneficios de corto plazo que representa ese alineamiento, continúa siendo una recomendación de fuerzas políticas y funcionarios diplomáticos. Sostenemos aquí que ese utilitarismo lleva principalmente a reforzar viejos lazos de dependencia y perpetuarlos, por lo tanto, alejan a esos países de conseguir mayores márgenes de autonomía.

A mediados de la década de 1990, David Blaney, en su trabajo "Reconceptualizing autonomy" (1996), hizo un gran aporte con la noción de autonomía relacional. Allí, sin abandonar el sentido y contenido inicial del concepto de autonomía en América Latina, sostuvo que: “[...] podemos llegar a ver la autonomía de las comunidades políticas como una especie de relación disponible en una sociedad global que apoya la autodeterminación y la autorrealización, no como una separación de los demás” (1996, p. 473). ${ }^{9}$

9 " $[\ldots]$ we can come to see the autonomy of political communities as a kind of relationship available in a global society supportive of self-determination and self-realization, not as separation from other". Traducción propia. 
Unos años después, Russell y Tokatlian (2001) tomaron ese concepto para repensar las estrategias de política internacional para los países latinoamericanos. Reflejando en la teoría los cambios globales de fines de siglo XX, vincularon la autonomía con los procesos de regionalización, democratización, globalización y relativo alejamiento de los Estados Unidos del escenario latinoamericano, dando especial preponderancia al rol de las instituciones para evitar caer en el "aislamiento" (2001, p. 88).

El punto central aquí es que la reinterpretación que realizan -lejos de la de Blaney que recupera el valor del "cuestionamiento ético" de la Teoría de la Dependencia (1996, p. 485)- parece desconocer la vigencia de las relaciones de poder y dominación en el sistema internacional que hacen que exista una clara distinción vigente entre países centrales, dominantes, y países débiles y dependientes (Míguez, 2013); aquella que dio origen y sentido también a la Doctrina de la 204 Autonomía. Si bien Russell y Tokatlian afirman considerar ese tipo de relaciones y evalúan que la autonomía relacional es la estrategia más eficaz en el nuevo contexto "para disminuir las asimetrías de poder y contrarrestar esas prácticas mediante una participación competente, activa, comprometida y responsable en los asuntos mundiales" (2001, p. 180), desconocen el necesario grado de confrontación que implica la construcción de una participación contrahegemónica en el sistema internacional (Míguez, 2013). Recuperamos aquí la noción de Blaney, y la importancia de aplicarla en especial a la región del Cono Sur. El exclusivismo nacional se muestra limitado en general, pero más aún en el caso de Estados pequeños, donde la apelación a la autonomía no puede remitirse, en muchos casos, a la construcción de un mercado interno ampliado de base nacional, porque las escalas lo dificultan -entre otros elementos-. Pero la importancia de la construcción de espacios regionales colectivos no puede soslayar que el ejercicio de autonomía en el contexto y la historia 
de los países dependientes implican confrontar con estructuras de poder establecidas. ${ }^{10}$

Un artículo reciente de Pinheiro y Lima (2018) reflexiona justamente sobre en qué medida los distintos "ajustes" conceptuales posteriores encierran una contradicción con el contenido inicial del concepto fruto de la experiencia histórica que le dio origen y sentido. En el mismo sentido desarrolla Míguez la noción de "autonomía con adjetivos" (2021, en prensa).

En cuanto al concepto que queremos rescatar, fue Mario Rapoport (2013) quien en el prólogo del libro de María Cecilia Míguez, Partidos Políticos y Política Exterior Argentina, se refirió a la noción de autonomía regional reinterpretando la noción de autora de la búsqueda de la autonomía en forma conjunta en el escenario latinoamericano.

Ahora bien, teniendo en cuenta los puntos de partida teóricos a los que hemos hecho referencia, ¿qué significa o

10 En Brasil, nuevos aportes respecto de la autonomía centraron su debate alrededor de las acciones tácticas relacionadas a la autonomía, más que a su caracterización. Desde el ámbito diplomático, Fonseca (1998) propuso discutir la búsqueda de autonomía, categorizándola a partir de sus acciones tácticas en momentos históricos dados como "autonomía por la distancia" y "autonomía por la participación". Posteriormente, Tullo Vigevani lideró una reflexión sistemática sobre el tema. Una de las derivaciones que tuvo el pensamiento sobre la autonomía es aquella que pondera la diversificación de las relaciones exteriores como elemento de peso para la construcción de márgenes de autonomía respecto de potencias tradicionales predominantes en la región. Vigevani y Cepaluni (2007), a partir de un pormenorizado análisis de la política exterior de los gobiernos de Lula en Brasil, realizaron una clasificación de lo que consideran variantes en la estrategia de autonomía que entienden como tradición histórica para ese país. La "autonomía por la distancia" supone una política de contestación a las normas y los principios de importantes instituciones y regímenes internacionales, vistos como favorables a mantener el statu quo, una tendencia autárquica y proteccionista a la hora de pensar el desarrollo. Por su vez, la "autonomía por la participación" cambia el posicionamiento frente a los regímenes internacionales, señalando la adhesión a los mismos como estrategia para influir dentro de ellos y, así, lograr los objetivos que el Estado se plantea. Mientras tanto, la "autonomía por la diversificación" supone la adhesión a los principios y las normas internacionales mediante las alianzas Sur-Sur, inclusive regionales, para reducir asimetrías y aumentar la capacidad de negociación frente a los países desarrollados, en un marco multipolar y evitando fenómenos autárquicos y rupturistas (Vigevani y Cepaluni, 2011, pp. 22-23). 
qué factores definen esa autonomía regional? Dicho de otro modo, ¿en qué casos los regionalismos, accionares políticos conjuntos y/o los procesos de integración son coconstitutivos de un proceso de autonomización de la política internacional de las unidades nacionales?

Como primera aproximación a la definición de este concepto, proponemos que existe mayor autonomía en función de un proceso de integración regional o regionalismo cuando:

- Se ven impulsadas políticas de reivindicación histórica del Estado pequeño, encarnadas en una interpretación histórica de un colectivo que se proyectan como fundamento de líneas de acción presentes y futuras

- Se recuperan, retoman o revalorizan tradiciones políticas e identitarias locales significativas por parte de fuerzas sociales y partidos políticos, entre otros, en el marco de la construcción de una identidad conjunta colectiva regional.

- Los intereses que se construyen políticamente fijan objetivos congruentes con la identidad de los agentes.

- Aumentan los cuestionamientos a las reglas de juego de organismos globales que garantizan las asimetrías del orden internacional.

- Hay modificaciones, como orientación estratégica, en cuanto a la relación con las potencias predominantes del sistema internacional que implican disminución de su injerencia en la toma de decisiones en distintos ámbitos, entre los que se destacan la política económica, la política de defensa y el desarrollo científico tecnológico.

- El proceso de integración impulsa la diversificación de vínculos económicos y políticos en el sistema internacional, promoviendo la existencia de nuevos aliados y socios. 
- Las políticas de integración refuerzan instituciones democráticas, protegen su funcionamiento, promueven políticas coordinadas de ampliación de derechos y mejoras en las condiciones de vida de las mayorías.

El concepto de autonomía relacional tiene la virtud de otorgar relevancia al vínculo con otros países como constitutivo de las propias capacidades y como instrumento para la consecución de objetivos. Refuerza la importancia de la construcción con otros frente a la confrontación. Sin embargo, desconoce el carácter contradictorio de las demandas de autonomía y el orden jerárquico global. La noción de una autonomía regional (Rapoport, 2013) o de una autonomía entre pares (Míguez, 2017) tiene la función de remarcar que dicha construcción colectiva prioriza socios en situación de simetría para cuestionar la distribución del poder. Ello no implica necesariamente subvertirla, pero sí al menos recortar el predominio que ejercen las potencias hegemónicas sobre los países dependientes. Se relaciona con las experiencias recientes en América Latina a las que Preciado Coronado (2014) calificó como nueva gramática democrática.

Una reconceptualización de la idea de autonomía regional, pensada desde los Estados pequeños, necesariamente demanda el reconocimiento de las asimetrías con los socios mayores de los procesos regionales. Como se verá en el último apartado, los costos de la apuesta regional tienen potencialidad de justificarse con los beneficios que la autonomía trae para el colectivo regional que posibilita una mayor agencia para este tipo de Estados.

\section{A modo de conclusión: del análisis histórico a la propuesta de un concepto de autonomía regional para Estados pequeños}

A la luz del análisis histórico buscamos desarrollar brevemente el devenir de la política uruguaya respecto de la 
integración regional. Dialogamos teóricamente con el paradigma de la autonomía para realizar una periodización y, así, abordar el rol que las dirigencias políticas le otorgaron a la región en cada gestión. Afirmamos que durante los gobiernos del FA existió una búsqueda de autonomía pero en una acepción particular, que remite a las características de un Estado pequeño. Por lo tanto, sostenemos que esa búsqueda de autonomía solamente pudo ser posible en el contexto de un ciclo progresista con capacidad de acción colectiva. En ese marco, las élites gobernantes compartieron un objetivo: buscar potenciar la capacidad de agencia para conseguir autonomía.

En términos globales, hubo un contexto de permisividad internacional. Luego del enorme y abrumador rechazo a las políticas neoliberales en toda la región, el siglo XXI se caracterizó por una economía en crecimiento en el contexto del ciclo de las commodities, el relativo alejamiento de los Estados 208 Unidos, el ascenso de China y la configuración de un Sur global que se visibilizaba como un espacio alternativo.

Este contexto favoreció a la constitución de viabilidad nacional para Estados pequeños en el marco de procesos regionales. Ahora bien, esos procesos regionales fueron objeto de disputa y de dilemas internos que los aquejan aún hoy, impidiendo su éxito.

La tensión entre las nociones de autonomía nacional y de integración regional constituye una constante en los períodos en que se fortalecen los mercados internos de los países más grandes del Cono Sur. Los costos de la integración son absolutamente palpables para los Estados más pequeños. Las salidas individuales o de alineamiento tradicional con potencias globales constituyen una constante vía rápida para el beneficio de estos últimos. ${ }^{11}$

\footnotetext{
11 Agradecemos los comentarios de Detlef Nolte y Peter Birle en oportunidad de una presentación preliminar de las ideas de este trabajo.
} 
Más autonomía puede implicar al mismo tiempo menos soberanía. Apelamos en este sentido a la definición conceptual que realizamos al inicio de nuestro trabajo. La autonomía es una estrategia y un objetivo. Remite a una tradición histórica latinoamericana que cuestiona la dependencia. Dichos lazos continúan vigentes en la situación actual, por ello, construir autonomía implica disminuirlos, es decir, no es solamente una capacidad estatal ni de las élites, es un contenido económico, social, político y cultural.

Si utilizamos una acepción estrecha de la autonomía, instrumental, podemos afirmar que un Estado pequeño puede decidir en forma "autónoma" una inserción liberalizante, que en ese caso controla su decisión y sus efectos. Sin embargo, las consecuencias de esa decisión, el entramado institucional y normativo que va gestando le va restringiendo capacidad de acción y margen de maniobra. En los hechos, está reforzando los lazos de dependencia con el sistema global. Por eso, aunque conlleve costos incluso en la soberanía, la integración a la que nos referimos implica facilitar la autodeterminación y la autorrealización de los proyectos políticos nacionales en sentido profundo.

Así la autonomía puede verse como medio y fin por parte de una constelación regional de actores políticos con identidades, mapas ideacionales compartidos que permiten una articulación razonable de sus políticas exteriores. Estas prácticas autonomistas ven la integración en clave solidaria. Es decir, a partir del predominio de lo político -que contiene en esencia al resto de dimensiones y especialmente a la socialsobre bases culturales compartidas en términos de valores.

En las tradiciones del realismo clásico y del realismo estructural, la autonomía aparece como un objetivo de todos los Estados (Harknett y Yalcin, 2012). Sin embargo, en nuestra perspectiva, que reivindica la importancia de la conceptualización históricamente situada (Cervo, 2008), autonomía y dependencia forman parte de una unidad dialéctica 
de sentido, porque la dependencia caracteriza a las formaciones económico-sociales latinoamericanas (Marini, 2008). Eso hace que el concepto de autonomía en América Latina adquiera un significado particular; está asociado profundamente al de interés nacional. La definición del interés nacional incluye elementos de larga y media duración, y coyunturales. Debe ser interpretado históricamente tal como lo ha desarrollado ampliamente la Historia de las Relaciones Internacionales, y la perspectiva crítica de Robert Cox (1981). En el caso de América Latina, el dominio colonial, la dependencia y las consecuencias vigentes de esas estructuras, signan su presente más allá de las transformaciones actuales del sistema internacional. Por lo tanto, ese interés nacional subyacente, reinterpretado por actores históricamente situados en contextos de disputa por los significados, relacionado con la disminución de los lazos de subordinación, resuena una y otra vez en distintas coyunturas. Eso es lo que hace que la 210 reflexión sobre la autonomía como concepto, como estrategia y como objetivo siga siendo tan relevante. En el caso de Uruguay, el Mercosur constituyó, bajo las condiciones antes señaladas, una pieza clave para brindar mayor posibilidad de acción autonómica para la política exterior uruguaya.

\section{Camilo López Burian}

Doctor en Ciencia Política (Universidad de la República Udelar). Profesor adjunto en la UdelaR (Ciencia PolíticaFacultad de Ciencias Sociales y Relaciones InternacionalesFacultad de Derecho). Integra el Sistema Nacional de Investigadores (ANII). Investiga sobre relaciones internacionales, políticas exteriores comparadas y derechas y política internacional.

\section{María Cecilia Míguez}

Doctora en Ciencias Sociales (FSOC-UBA), Investigadora Adjunta de CONICET. Es Vice directora del Instituto 
de Investigaciones Históricas Económicas Sociales e Internacionales (IDEHESI), docente de grado y posgrado. Autora de libros y numerosos artículos sobre política internacional argentina.

\section{Bibliografía}

ACUÑA, Carlos Hugo; CHUDNOVSKY, Mariana. 2013. Cómo entender las instituciones y su relación con la política: lo bueno, lo malo y lo feo de las instituciones y los institucionalismos. In: ACUÑA, Carlos Hugo (ed.). ¿Cuánto importan las instituciones? Gobierno, Estado y actores en la política argentina. Buenos Aires: Siglo XXI. pp. 13-67.

BALDACCHINO, Godfrey; WIVEL, Anders. 2020. Small states: concepts and theories. In: BALDACCHINO, Godfrey; WIVEL, Anders (ed.). Handbook on the politics of small states. Cheltenham: Edward Elgar Publishing. pp. 2-19.

BERNAL-MEZA, Raúl. 2005. América Latina en el mundo: el pensamiento latinoamericano y la teoría de relaciones internacionales. Buenos Aires: Grupo Editor Latinoamericano.

BERNAL-MEZA, Raúl. 2016. Contemporary Latin American thinking on international relations: theoretical, conceptual and methodological contributions. Revista Brasileira de Política Internacional, v. 59, n. 1, pp. 1-32.

BLANEY, David L. 1996. Reconceptualizing autonomy: the difference dependency theory makes. Review of International Political Economy, v. 3, n. 3, pp. 459-497.

BOLOGNA, Alfredo Bruno. 2008. La vigencia de la teoría de la autonomía de Puig para analizar el gobierno de Menem. Ágora Internacional, v. 3, n. 6, pp. 25-34.

BRICEÑO RUIZ, José. 2012. Autonomía y desarrollo en el pensamiento integracionista latinoamericano. In: BRICEÑO RUIZ, José; RIVAROLA PUNTIGLIANO, Andrés; CASAS GRAGEA, Ángel María (ed.). Integración latinoamericana y caribeña: politica y economía. Madrid: Fondo de Cultura Económica. pp. 27-58.

BRICEÑO RUIZ. José. 2015. Saber y teoría: reconstruyendo la tradición autonómica en los estudios de integración en América Latina. In: BRICEÑO RUIZ, José; SIMONOFF, Alejandro (ed.). Integración y cooperación regional en América Latina: una relectura a partir de la teoría de la autonomía. Buenos Aires: Editorial Biblos. pp. 29-69. 
BRICEÑO RUIZ, José; SIMONOFF, Alejandro (ed.). 2015. Integración y cooperación regional en América Latina: una relectura a partir de la teoría de la autonomía. Buenos Aires: Editorial Biblos.

CAETANO, Gerardo; LÓPEZ BURIAN, Carlos; LUJÁN, Carlos. 2016. La política exterior uruguaya entre Vázquez y Vázquez (2010-2015). In: BUSQUETS, José; BENTANCUR, Nicolás (coord.). El decenio progresista: las políticas públicas de Vázquez a Mujica. Montevideo: Fin de Siglo. pp. 279-300.

CEPEDA ULLOA, Fernando. 1986. La lucha por la autonomía: la gran encrucijada de la política exterior de Betancur. In: MUÑOZ, Heraldo (comp.). América Latina y el Caribe: políticas exteriores para sobrevivir. Buenos Aires: Grupo Editor Latinoamericano. pp. 209-236.

CERVO, Amado Luiz. 2008. Inserção internacional: formação dos conceitos brasileiros. São Paulo: Editora Saraiva.

COLACRAI, Miryam. 2009. Los aportes de la teoría de la autonomía, genuina contribución sudamericana. ¿La autonomía es hoy una categoría en desuso o se enfrenta al desafío de una renovación en un contexto interdependiente y más complejo? In: LECHINI, Gladys; KLAGSBRUNN, Victor; GONÇALVES, William (org.). Argentina e Brasil: vencendo os preconceitos: as várias arestas de uma concepção estratégica.

COX, Robert W. 1981. Social forces, states and world orders: beyond international relations theory. Millennium: Journal of International Studies, v. 10, n. 2, pp. 126-155.

DALLANEGRA PEDRAZA, Luis. 2009. Realismo-sistémico-estructural: politica exterior como "construcción" de poder. Córdoba: Edición del autor.

DECIANCIO, Melisa. 2016. El regionalismo latinoamericano en la agenda de relaciones internacionales. Iberoamericana. América Latina-EspañaPortugal, v. 16, n. 63, pp. 91-110.

DREKONJA KORNAT, Gerhard. 1983. Retos de la politica exterior colombiana. Bogotá: CEREC-CEI.

DREKONJA KORNAT, Gerhard. 1993. Autonomía periférica redefinida: América Latina en la década de los noventa. Bogotá: Universidad de los Andes. (Serie Documentos Ocasionales del Centro de Estudios Internacionales, n. 31, pp. 10-21).

ESCUDÉ, Carlos. 1992. Realismo periférico: fundamentos para la nueva politica exterior Argentina. Buenos Aires: Planeta.

ESCUDÉ, Carlos. 1995. El realismo de los estados débiles. Buenos Aires: Grupo Editor Latinoamericano.

FONSECA JÚNIOR, Gelson. 1998. A legitimidade e outras questões internacionais: poder e ética entre as nações. São Paulo: Paz e Terra. 
GARCÉ, Adolfo. 2014a. ¿Réquiem para la derecha? La elección de 2014 y la izquierda que se viene. In: GARCÉ, Adolfo; YAFFÉ, Jaime. La era progresista: hacia una nueva estrategia de desarrollo. Montevideo: Fin de Siglo. pp. 203-246.

GARCÉ, Adolfo. 2014b. Regímenes políticos de conocimiento: construyendo un nuevo concepto a partir de eventos seleccionados en políticas públicas del gobierno de Tabaré Vázquez (Uruguay, 20052009). Revista de Ciencia Política (Santiago), v. 34, n. 2, pp. 439-458.

GARCÉ, Adolfo; LÓPEZ BURIAN, Camilo. 2014. La politica exterior como política pública: Ideas, intereses e instituciones. Debates teóricos recientes desde la Ciencia Política. Paper presentado en la Conferencia FLACSO-ISA, Buenos Aires, 23 al 25 de julio.

HARKNETT, Richard J.; YALCIN, Hasan B. 2012. The struggle for autonomy: a realist structural theory of international relations. International Studies Review, v. 14, n. 4, pp. 499-521.

HERNÁNDEZ NILSON, Diego; LÓPEZ BURIAN, Camilo. 2020. Uruguay in the pandemic amid the return of the right: becoming an enclave. Latin American Policy, v. 11, n. 2, pp. 327-334.

JAGUARIBE, Helio. 1969. Dependencia y autonomía en América Latina. In: JAGUARIBE, Helio et al. (ed.). La dependencia político-económica de América Latina. México: Siglo XXI. pp. 1-85.

JAGUARIBE, Helio. 1979. Autonomía periférica y hegemonía céntrica. Estudios Internacionales, año 12, n. 46, pp. 91-130.

JAGUARIBE, Helio. (1982). Hegemonía céntrica y autonomía periférica. In: Hill, Eduardo; Tomassini, Luciano (comp.). América Latina y el nuevo orden económico internacional. Buenos Aires: Editorial Belgrano. pp. 17-48.

LECHINI, Gladys. 2009. La cooperación Sur-Sur y la búsqueda de autonomía en América Latina: ¿Mito o realidad? Relaciones Internacionales, n. 12, pp. 55-81.

LÓPEZ BURIAN, Camilo. 2015. Partidos politicos y politica exterior en Uruguay (1985-2015). La importancia de las instituciones, las ideas y los intereses de los actores. Tesis de Doctorado en Ciencia Política. Montevideo: ICP-FCS-UdelaR.

LÓPEZ BURIAN, Camilo. 2017. El consenso de los partidos Colorado y Nacional en la política exterior uruguaya (1985-2005). Revista de la Facultad de Derecho, n. 42, pp. 100-137.

LUJÁN, Carlos. 1993. Cambio de régimen y política internacional: el caso uruguayo. Montevideo: División Cultura, Intendencia Municipal de Montevideo. 
MARINI, Ruy. Mauro. 2008 [1973]. Dialéctica de la dependencia. In: MARTINS, Carlos Eduardo (ed.). América Latina, dependencia y globalización. Fundamentos conceptuales. Bogotá: Siglo del HombreCLACSO. pp. 107-149.

MÍGUEZ, María Cecilia. 2013. Partidos políticos y política exterior argentina. Buenos Aires: Ariel.

MÍGUEZ, María Cecilia. 2017. La autonomía heterodoxa y la clasificación de las políticas exteriores en la Argentina. Revista de Relaciones Internacionales, Estrategia y Seguridad, v. 12, n. 2, pp. 207-229.

MÍGUEZ, María Cecilia. 2021. La autonomía: un aporte latinoamericano a la teoría de las relaciones internacionales. In: ALVAREZ FUENTES, Gonzalo et al. (ed.) Teoría y práctica de las relaciones internacionales en América Latina. Santiago: Universidad Arturo Prat. En prensa.

MÍGUEZ, María Cecilia; DECIANCIO, Melisa Andrea. 2016. La internacionalización de la teoría de las relaciones internacionales en la Argentina: los híbridos teóricos y su clasificación. Papeles de Trabajo, v. 10, n. 18, pp. 169-192.

MINISTERIO DE RELACIONES INTERNACIONALES Y CULTO DE LA REPÚBLICA ARGENTINA. 2003. Consenso de Buenos Aires. Disponible en: https://bit.ly/3tWLdJh. Acceso en: 27 abr. 2021.

MINISTERIO DE TRABAJO DE LA REPÚBLICA ARGENTINA. 2004. Acta de Copacabana. Disponible en: https://bit.ly/3vlmw9G. Acceso en: 27 abr. 2021.

MIRANDA, Roberto Alfredo. 2003. Argentina: autonomía en tiempos de crisis. Relaciones Internacionales, v. 12, n. 24, pp. 127-140.

MIRANDA, Roberto Alfredo. 2005. Sobre los fundamentos internacionales de la política Argentina: teoría y realidad. Invenio: Revista de Investigación académica, v. 8, n. 15, pp. 47-60.

MONIZ BANDEIRA, Luiz Alberto. 2002. Las relaciones en el Cono Sur: iniciativas de integración. In: RAPOPORT, Mario; CERVO, Amado Luiz (comp.) El Cono Sur: una historia común. Buenos Aires: Fondo de Cultura Económica. pp. 281-322.

PALTI, Elías J. 2007. La nueva historia intelectual y sus repercusiones en América Latina. História Unisinos, v. 11, n. 3, pp. 297-305.

PERROTTA, Daniela; PORCELLI, Emanuel. 2019. El regionalismo es lo que la academia hace de él. Revista Uruguaya de Ciencia Política, v. 28, n. 1, pp. 183-218.

PINHEIRO, Letícia; LIMA, Maria Regina Soares de. 2018. Between autonomy and dependency: the place of agency in Brazilian foreign policy. Brazilian Political Science Review, v. 12, n. 3, e0003. 
PIZZORNO, Alessandro. 1985. Sobre la racionalidad de la opción democrática. In: CALDERÓN, Fernando (comp.). Los límites de la democracia. Buenos Aires: CLACSO. 2 v. pp. 13-52.

POSE, Nicolás. 2018. Ideas y política exterior económica en el mundo en desarrollo. El caso de la salida de Uruguay de las negociaciones del TiSA. Desafios, v. 30, n. 2, pp. 89-126.

PRECIADO CORONADO, Jaime Antonio. 2014. La nueva gramática democrática frente a la integración autónoma latinoamericana y caribeña. Sociedade e Estado, v. 29, n. 1, pp. 45-72.

PUIG, Juan Carlos. 1980. Doctrinas internacionales y autonomía latinoamericana. Caracas: Universidad Simón Bolívar.

PUIG, Juan Carlos. 1986. Integración y autonomía de América Latina en las postrimerías del siglo XX. Revisa Integración Latinoamericana, n. 109, pp. $40-62$.

QUILICONI, Cintia; RIVERA, Renato. 2019. Ideología y liderazgo en la cooperación regional: los casos del Consejo Suramericano de defensa y el Consejo Suramericano sobre el problema mundial de las drogas en Unasur. Revista Uruguaya de Ciencia Política, v. 28, n. 1, pp. 219-248.

QUILICONI, Cintia; SALGADO ESPINOZA, Raúl. 2017. Latin American integration: regionalism à la carte in a multipolar world? Colombia Internacional, n. 92, pp. 15-41.

RAPOPORT, Mario. 2013. Prólogo. In: MÍGUEZ, María Cecilia. Partidos políticos y política exterior argentina. Buenos Aires: Ariel. pp. 13-20.

ROSECRANCE, Richard. 1986. The rise of the trading state: commerce and conquest in the modern world. New York: Basic Books.

RUSSELL, Roberto. 1994. Los ejes estructurantes de la política exterior argentina. Revista América Latina/Internacional, v. 1, n. 2, pp. 5-26.

RUSSELL, Roberto; TOKATLIAN, Juan Gabriel. 2001. De la autonomía antagónica a la autonomía relacional: una mirada teórica desde el Cono Sur. Revista PostData, v. 7, n. 1, pp. 71-92.

SADER, Emir (org.). 2013. 10 anos de governos pós-neoliberais no Brasil: Lula e Dilma. São Paulo: Boitempo; Rio de Janeiro: Flacso.

SANAHUJA, José Antonio. 2008. Del "regionalismo abierto” al "regionalismo post-liberal". Crisis y cambio en la integración regional en América Latina. In: MARTÍNEZ ALFONSO, Laneydi; PEÑA, Lázaro; VAZQUEZ, Mariana (coord.). Anuario de la integración regional de América Latina y el Gran Caribe 2008-2009. Buenos Aires: Cries. pp. 11-54.

SANAHUJA, José Antonio. 2014. Enfoque diferenciados y marcos comunes en el regionalismo latinoamericano: alcance y perspectivas de UNASUR y CELAC. Pensamiento Propio, v. 19, pp. 75-108. 
SANAHUJA, José Antonio. 2018. Reflexividad, emancipación

y universalismo: cartografías de la teoría de las relaciones internacionales. Revista Española de Derecho Internacional, v. 70, n. 2, pp. 101-125.

SANAHUJA, José Antonio. 2019. Crisis de la globalización, el regionalismo y el orden liberal: el ascenso mundial del nacionalismo y la extrema derecha. Revista Uruguaya de Ciencia Política, v. 28, n. 1, pp. 59-94.

SANAHUJA, José Antonio; LÓPEZ BURIAN, Camilo. 2020.

Internacionalismo reaccionario y nuevas derechas neopatriotas latinoamericanas frente al orden internacional liberal. Conjuntura Austral, v. 11, n. 55, pp. 22-34.

SIMONOFF, Alejandro. 2007. Teoría y práctica de la autonomía: la política exterior de Illia. Relaciones Internacionales, v. 16, n. 32, pp. 1-18. SIMONOFF, Alejandro. 2010. La Argentina y el mundo frente al bicentenario de la Revolución de Mayo: las relaciones exteriores argentinas desde la secesión de España hasta la actualidad. La Plata: EDULP.

SIMONOFF, Alejandro. 2013. Dilemas históricos y presentes de nuestro relacionamiento externo. In: SÁNCHEZ, Leandro et al. Reflexiones sobre 216 política exterior. La Plata: UNLP. pp. 87-94.

SIMONOFF, Alejandro. 2016. La escuela autonomista: pasado y presente. Perspectivas. Revista de Ciencias Sociales, v. 1, pp. 9-19.

SIMONOFF, Alejandro; LORENZINI, María Elena. 2019. Autonomía e integración en las teorías del sur: desentrañando el pensamiento de Hélio Jaguaribe y Juan Carlos Puig. Iberoamericana: Nordic Journal of Latin American and Caribbean Studies, v. 48, n. 1, pp. 96-106.

TOMASSINI, Luciano. 1989. Teoría y práctica de la política internacional. Santiago: Ediciones Universidad Católica de Chile.

TUSSIE, Diana. 2009. Latin America: contrasting motivations for regional projects. Review of International Studies, v. 35, pp. 169-188. Suplemento 1.

VIGEVANI, Tullo; CEPALUNI, Gabriel. 2007. A política externa de Lula da Silva: a estratégia da autonomia pela diversificação. Contexto Internacional, v. 29, n. 2, pp. 273-335.

VIGEVANI, Tullo; CEPALUNI, Gabriel. 2011. A política externa brasileira: a busca da autonomia, de Sarney a Lula. São Paulo: Editora Unesp.

WENDT, Alexander. 1992. Anarchy is what states make of it: the social construction of power politics. International Organization, v. 46, n. 2, pp. 391-425. 


\section{URUGUAY COMO ESTADO PEQUEÑO EN EL MERCOSUR (1991-2020): UNA LECTURA DESDE LA AUTONOMÍA REGIONAL}

\section{CAMILO LÓPEZ BURIAN}

MARÍA CECILIA MÍGUEZ

Resumen: Desde una perspectiva histórica, este trabajo caracteriza diferentes etapas de la política exterior de Uruguay frente al Mercosur. Dialoga teóricamente con el paradigma de la autonomía y sostiene que durante los gobiernos de izquierda existió una búsqueda de autonomía pero en una acepción particular, que remite a las características de un Estado pequeño. Esa búsqueda de autonomía solamente pudo ser posible en el contexto de un ciclo progresista con capacidad de acción colectiva. En ese marco, las élites gobernantes compartieron un objetivo: buscar potenciar la capacidad de agencia para conseguir autonomía. Como conclusión se presenta un concepto de autonomía regional que propone el carácter coconstitutivo de la autonomía y la integración regional, pensado desde el caso uruguayo como Estado pequeño.

Palabras Clave: Uruguay; Estados Pequeños; Mercosur; Regionalismo; Integración Regional; Autonomía Regional.

\section{URUGUAI COMO PEQUENO ESTADO NO MERCOSUL (1991-2020): UMA LEITURA A PARTIR DA AUTONOMIA REGIONAL}

Resumo: A partir de uma análise histórica, este trabalho caracteriza as diferentes etapas da politica externa do Uruguai em relação ao Mercosul. Dialogando teoricamente com o paradigma da autonomia, propõe que durante os governos de esquerda houve uma busca pela autonomia, mas num sentido particular, que remete às características de um pequeno Estado. Essa busca de autonomia só foi possível no contexto de um ciclo 
progressista com capacidade de ação coletiva. Nesse contexto, as elites dominantes compartilhavam um objetivo: buscar aumentar a capacidade da agência para alcançar a autonomia. Como conclusão, apresenta um conceito de autonomia regional que propõe o caráter co-constitutivo da autonomia e da integração regional, pensada a partir do caso uruguaio como um pequeno Estado.

Palavras-chave: Uruguai; Pequenos Estados; Mercosul; Regionalismo; Integração Regional; Autonomia Regional.

\section{URUGUAY AS A SMALL STATE IN MERCOSUR (1991-2020): A READING FROM REGIONAL AUTONOMY}

Abstract: This paper characterizes the different stages of Uruguay's foreign policy towards Mercosur (1991-2021), with a historical perspective. It dialogues theoretically with the autonomy paradigm and argues that during the leftist governments autonomy became an objective, with a particular meaning, linked to the characteristics of a small state. The search for autonomy was possible in the context of a progressive cycle and the capacity for collective action. In that period, unlike the others, the ruling elites shared one objective: to enhance agency in a collective way. As a conclusion, we present a concept of regional autonomy that proposes the co-constitutional character of autonomy and regional integration, thought of from the Uruguayan case as a small State.

Keywords: Uruguay; Small States; Mercosur; Regionalism; Regional Integration; Regional Autonomy.

Recebido: 05/01/2021Ａprovado: 23/03/2021 